\title{
ANÁLISE DA EXPOSIÇÃO A FATORES DE RISCO EXTRÍNSECOS ASSOCIADOS AO ENVELHECIMENTO DA PELE EM UNIVERSITÁRIOS
}

\author{
ANÁLISIS DE LA EXPOSICIÓN A FACTORES DE RIESGO EXTRÍNSECOS \\ ASOCIADOS AL ENVEJECIMIENTO CUTÁNICO EN ESTUDIANTES \\ UNIVERSITARIOS
}

\section{ANALYSIS OF EXPOSURE TO RISK FACTORS ASSOCIATED WITH SKIN AGING IN UNIVERSITY STUDENTS}

\author{
Maria Clara Olival SANTOS ${ }^{1}$ \\ Sabrina Oliveira ELIZEU ${ }^{2}$ \\ Lucivânia Mota MATOS ${ }^{3}$ \\ Graciela dos Santos SOARES ${ }^{4}$ \\ Silmara Patrícia Correia da Silva MACRI ${ }^{5}$ \\ Luiz Henrique da Silva NALI ${ }^{6}$
}

RESUMO: O envelhecimento é inevitável, dentre os diversos órgãos que sofrem com o envelhecimento, a pele é um órgão que sofre consideravelmente com fatores externos, o que potencialmente interfere no envelhecimento precoce do órgão. Tal envelhecimento está associado a uma maior probabilidade de desenvolvimento de doenças dermatológicas bem como interfere substancialmente em questões estéticas. Com base no suposto, o objetivo desse estudo foi o de analisar a frequência de exposição de universitários a fatores de risco envolvidos com o envelhecimento da pele. Foram convidados a participar do estudo universitários de diversos cursos da área da saúde. Cada voluntário respondeu a um questionário estruturado contendo perguntas relacionadas a exposição a fatores de risco relacionados ao envelhecimento cutâneo, como tabagismo, etilismo, exposição prolongada ao sol, ausência de foto proteção e poucas horas de sono por dia. Ao todo foram entrevistados 298 voluntários de diversos cursos da área da saúde, sendo que a maioria se declarou da etnia branca. Encontramos uma baixa frequência do uso do cigarro (11\%), porém frequência mais elevada de indivíduos que ingeriam álcool (42\%), e adicionalmente cerca de $33 \%$ dos indivíduos relataram exposição ao sol, e cerca de $56 \%$ dos entrevistados não faziam uso de foto proteção. Em relação ao sono, cerca de $56 \%$ dos indivíduos descreveram ter menos do que 6 horas de sono diárias. A população estudada

\footnotetext{
${ }^{1}$ Universidade Santo Amaro (UNISA), São Paulo - SP - Brasil. Graduanda no Curso de Estética e Cosmética. ORCID: https://orcid.org/0000-0002-6545-8456. E-mail: maria13olival2000@gmail.com

${ }^{2}$ Universidade Santo Amaro (UNISA), São Paulo - SP - Brasil. Graduanda no Curso de Estética e Cosmética. ORCID: https://orcid.org/0000-0001-8449-3591. E-mail: sabrinasasa0902@gmail.com

${ }^{3}$ Universidade Santo Amaro (UNISA), São Paulo - SP - Brasil. Graduanda no Curso de Estética e Cosmética. ORCID: https://orcid.org/0000-0002-4013-0403. E-mail: vaniadamottaemattos@gmail.com

${ }^{4}$ Universidade Santo Amaro (UNISA), São Paulo - SP - Brasil. Mestranda no Programa de Pós-graduação em Ciências da Saúde. ORCID: https://orcid.org/0000-0003-4627-1538. E-mail: gracielabonani@gmail.com

${ }^{5}$ Universidade Santo Amaro (UNISA), São Paulo - SP - Brasil. 3. Docente dos cursos de Fisioterapia e Estética. Mestrado em Engenharia Biomédica (UMC). ORCID: https://orcid.org/0000-0001-6864-0360. E-mail: silmaraft15@gmail.com

${ }^{6}$ Universidade Santo Amaro (UNISA), São Paulo - SP - Brasil. 2. Docente dos cursos de Fisioterapia e Estética. Doutorado em Medicina Tropical (USP). ORCID: https://orcid.org/0000-0002-8365-9796. E-mail: lnali@prof.unisa.br
} 
está frequentemente exposta aos principais fatores intrínsecos e extrínsecos envolvidos com o envelhecimento cutâneo.

PALAVRAS-CHAVE: Envelhecimento cutâneo. Universitários. Sono. Cigarro. Álcool.

RESUMEN: El envejecimiento es inevitable, entre los diversos órganos que sufren el envejecimiento, la piel es un órgano que sufre considerablemente por factores externos, lo que potencialmente interfiere con el envejecimiento prematuro del órgano. Dicho envejecimiento está asociado con una mayor probabilidad de desarrollar enfermedades dermatológicas, además de interferir sustancialmente con los problemas estéticos. Partiendo del supuesto, el objetivo de este estudio fue analizar la frecuencia de exposición de los estudiantes universitarios a los factores de riesgo relacionados con el envejecimiento cutáneo. Se invitó a participar del estudio a estudiantes universitarios de diferentes cursos de salud. Cada voluntario respondió a un cuestionario estructurado que contenía preguntas relacionadas con la exposición a factores de riesgo relacionados con el envejecimiento cutáneo, como fumar, beber, exposición prolongada al sol, ausencia de fotoprotección y pocas horas de sueño al día. En total, se entrevistaron 298 voluntarios de diferentes cursos de salud, la mayoría de los cuales se declararon blancos. Encontramos una baja frecuencia de consumo de cigarrillos (11\%), pero una mayor frecuencia de personas que bebían alcohol (42\%) y, además, alrededor del $33 \%$ de las personas informaron haber estado expuestas al sol y aproximadamente el $56 \%$ de los encuestados no consumieron fotoprotección. Con respecto al sueño, alrededor del 56\% de las personas informaron tener menos de 6 horas de sueño al día. La población estudiada está frecuentemente expuesta a los principales factores intrínsecos y extrínsecos implicados en el envejecimiento cutáneo.

PALABRAS CLAVE: Envejecimiento cutáneo. Estudiantes universitarios. Dormir. Cigarrillo. Alcohol.

ABSTRACT: Aging is inevitable, among the several organs that suffer with aging, the skin is an organ that suffers considerably with external factors, which potentially interferes on the precocious aging of the organ. Such aging is associated with a higher probability of developing dermatological diseases as well as substantially interferes with aesthetic issues. Based on this assumption, the objective of this study was to analyze the frequency of exposure of university students to risk factors involving skin aging. Were invited to participate in the study students of several university courses in the area of health. Each volunteer answered a structured questionnaire containing questions related to exposure to risk factors related to skin aging, such as smoking, alcoholism, prolonged sun exposure, absence of photo protection and few hours of sleep per day. In all, 298 volunteers from various health courses were interviewed, most of whom declared themselves white. We found a low frequency of smokers (11\%), but higher frequency of individuals who ingested alcohol (42\%), and additionally about 33\% of individuals reported sun exposure, and about $56 \%$ of respondents did not use photo protection. Regarding sleep, about 56\% of the individuals described having less than 6 hours of sleep per day. The studied population is frequently exposed to the main intrinsic and extrinsic factors involved with skin aging.

KEYWORDS: Cutaneous aging. College students. Sleep. Cigarette. Alcohol. 


\section{Introdução}

A pele é o maior órgão do corpo humano, composta por uma diversidade celular considerável e apresenta função fundamental na proteção do organismo contra desidratação, câncer, atritos, agentes infecciosos, bem como apresenta papel fundamental na homeostase do organismo. Infelizmente, o processo de envelhecimento cutâneo é inevitável e mudanças consideráveis ocorrem na questão estrutural e funcional da pele (BONTÉ et al., 2019). Esse envelhecimento pode ser acelerado por fatores intrínsecos, como distúrbios metabólicos, por exemplo o diabetes (TOBIN, 2017), e extrínsecos, como exposição prolongada e desprotegida ao sol, tabagismo, etilismo, poluição, além fatores relacionados a uma melhor qualidade de vida como uma boa alimentação e um período ideal de sono (YOUNG, 2006; YIN et al., 2001; KRUTMANN et al., 2017).

Do ponto de vista fisiopatológico as principais alterações estruturais que ocorrem na pele são fruto de efeitos oxidativos que induzem alterações estruturais no colágeno e na elastina, o que consequentemente afeta a composição da matriz extracelular do tecido conjuntivo, ou ainda a mudanças a nível de DNA, bem como afeta a função transportadora da membrana celular (KAMMEYER; LUITEN, 2015). Tais mudanças levam a importante perda da função fisiológica da pele, bem como modificações estruturais características do envelhecimento. Sabidamente, esse envelhecimento cutâneo precoce pode apresentar impactos consideráveis na autoestima das pessoas, bem como em sua qualidade de vida, além disso apresenta riscos para o desenvolvimento de cânceres cutâneos, como o melanoma. Sendo assim, o objetivo desse estudo foi de analisar a frequência de exposição aos principais fatores de risco extrínsecos ao envelhecimento cutâneo.

\section{Métodos}

O desenho desse trabalho teve caráter observacional sem intervenções e com aplicação de um questionário estruturado com 7 perguntas para averiguar a frequência de exposição aos principais fatores de risco extrínsecos ligados ao envelhecimento cutâneo. O estudo foi conduzido em uma universidade particular situada na zona sul do município de São Paulo. Foram convidados a participar do estudo estudantes dos cursos da área da saúde de 18 a 40 anos de ambos os gêneros. Os voluntários responderam o questionário após a leitura e assinatura do Termo de Consentimento Livre e Esclarecido (TCLE). O presente estudo foi aprovado pelo Comitê de Ética e Pesquisa da Universidade Santo Amaro, com o CAAE \#19033019.6.0000.0081. 
As diferentes variáveis foram tabuladas e analisadas com relação a sua significância perante os principais fatores de risco, no intuito de obter aqueles fatores mais prevalentes em universitários. Sendo assim, os dados foram de análises com base no teste Wilcox e as variáveis contínuas utilizamos o teste de anova.

\section{Resultados}

Ao todo, foram entrevistados 298 acadêmicos dos cursos de Fisioterapia, Estética e Cosmética, Ciências Biológicas, Educação Física e Odontologia. Houve uma predominância de indivíduos do sexo feminino (61.4\%), participando do estudo, em relação a indivíduos do sexo masculino (38.6\%). A população do estudo foi predominantemente jovem, com idade média variando de 21.7 a 24.9 anos entre os cursos avaliados, e uma parcela considerável dos pacientes incluídos no estudo declararam-se brancos, seguidos de pardos e negros. Os dados demográficos dos voluntários participantes do estudo estão descritos na tabela 1 .

Tabela 1 - Dados demográficos dos voluntários incluídos no estudo

\begin{tabular}{ccccccc}
\hline Variável(n) & $\begin{array}{c}\text { Fisioterapia } \\
\mathbf{( 4 9 )}\end{array}$ & $\begin{array}{c}\text { Ciências } \\
\text { Biológicas } \\
(\mathbf{5 0}\end{array}$ & $\begin{array}{c}\text { Farmácia } \\
\mathbf{( 5 0 )}\end{array}$ & $\begin{array}{c}\text { Estética } \\
\mathbf{( 5 0 )}\end{array}$ & $\begin{array}{c}\text { Odontologia } \\
\mathbf{( 5 0 )}\end{array}$ & $\begin{array}{c}\text { Educação } \\
\text { Física (49) }\end{array}$ \\
\hline $\begin{array}{c}\text { Idade } \\
(\mathbf{m e ́ d i a )}\end{array}$ & $17-56(24)$ & $17-44(21,7)$ & $\begin{array}{c}17-65 \\
(27,7)\end{array}$ & $\begin{array}{c}17-45 \\
(22,8)\end{array}$ & $17-62(24,9)$ & $17-36$ \\
$\begin{array}{c}\text { Homens (\%) } \\
\text { Mulheres }\end{array}$ & $37(75,5)$ & $16(32)$ & $21(42)$ & $1(2)$ & $8(16)$ & $32(65,3)$ \\
$(\%)$ & $12(24,5)$ & $34(68)$ & $29(58)$ & $49(98)$ & $42(84)$ & $17(34,7)$ \\
& $\mathrm{B}: 20$ & $\mathrm{~B}: 29$ & $\mathrm{~B}: 21$ & $\mathrm{~B}: 20$ & $\mathrm{~B}: 27$ & $\mathrm{~B}: 19$ \\
Cor da pele* & $\mathrm{P}: 14$ & $\mathrm{P}: 16$ & $\mathrm{P}: 15$ & $\mathrm{P}: 16$ & $\mathrm{P}: 18$ & $\mathrm{P}: 17$ \\
& $\mathrm{~N}: 15$ & $\mathrm{~N}: 5$ & $\mathrm{~N}: 11$ & $\mathrm{~N}: 9$ & $\mathrm{~N}: 4$ & $\mathrm{~N}: 10$ \\
\hline
\end{tabular}

* autodeclarada; $\mathrm{B}=\mathrm{Branco} ; \mathrm{P}=\mathrm{Pardo} ; \mathrm{N}=$ Negro

Fonte: Elaborado pelos autores

Avaliamos a frequência de pessoas que se declaravam etilistas ou tabagistas, as entrevistas revelaram que grande parte das pessoas afirmaram não fazer uso do tabaco. Com relação a prática do etilismo, uma proporção maior que a dos tabagistas alegava fazer ingestão de bebida alcoólica pelo menos 2 vezes na semana, conforme descrito na figura 1 . 
Figura1 - Relação dos indivíduos fumantes e etilistas

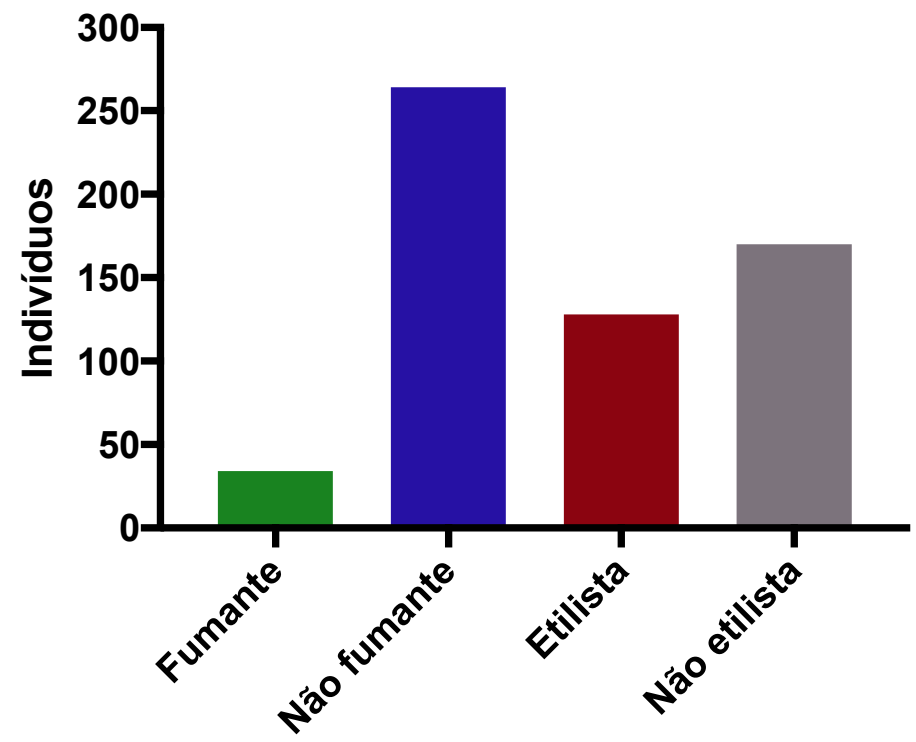

Fonte: Acervo dos autores

Um outro fator analisado pelo nosso grupo foi a duração do sono dos indivíduos de cada curso de uma maneira geral e o que foi observado, de acordo com a tabela 2 , é que grande parte dos estudantes apresentam 6 horas diárias ou menos de sono, o que foi estatisticamente significativo quanto comparado a indivíduos que dormem pelo menos 7 horas $(\mathrm{p}<0.01)$.

Tabela 2 - Horas de sono dos indivíduos para cada um dos cursos.

\begin{tabular}{lccccccccc}
\hline & \multicolumn{8}{c}{ Quantidade de horas de sono } \\
\cline { 2 - 10 } & $\mathbf{3 h}$ & $\mathbf{4 h}$ & $\mathbf{5 h}$ & $\mathbf{6 h}$ & $\mathbf{7 h}$ & $\mathbf{8 h}$ & $\mathbf{9 h}$ & $\mathbf{1 0 h}$ & $\mathbf{1 1 h}$ \\
\hline Ciências Biológicas & - & 5 & 10 & 14 & 7 & 12 & 3 & 2 & - \\
Educação Física & - & 1 & 9 & 15 & 12 & 7 & 1 & 2 & - \\
Estética e Cosmética & 1 & - & 9 & 14 & 3 & 15 & 5 & 2 & - \\
Farmácia & 3 & 8 & 13 & 16 & 5 & 4 & - & - & 1 \\
Fisioterapia & - & 5 & 9 & 12 & 10 & 8 & 5 & 1 & - \\
Odontologia & 1 & 7 & 3 & 13 & 7 & 8 & 1 & - & - \\
\hline Total & $\mathbf{5}$ & $\mathbf{2 6}$ & $\mathbf{5 3}$ & $\mathbf{8 3}$ & $\mathbf{4 5}$ & $\mathbf{5 4}$ & $\mathbf{1 5}$ & $\mathbf{7}$ & $\mathbf{1}$ \\
\hline
\end{tabular}

Fonte: Elaborado pelos autores

Adicionalmente, indagamos os universitários a respeito da exposição à luz solar e se eles usam foto proteção diária. De acordo com a figura 2 pudemos observar um dado preocupante, onde cerca de $33 \%$ dos indivíduos relataram exposição a raios solares em períodos de maiores incidência da luz solar, enquanto cerca de 56\% de todos os indivíduos não fazem uso de fotoprotetor. De todos os cursos observados, apenas os voluntários do curso de estética 
apresentaram taxa de uso de fotoprotetor de $66 \%$, seguido de Odontologia (48\%), Ciências biológicas (39,5\%), Fisioterapia (38\%), Farmácia (36\%) e Educação Física (34\%).

Figura 2 - Relação de indivíduos que relatam exposição ao sol e que utilizam fotoprotetor

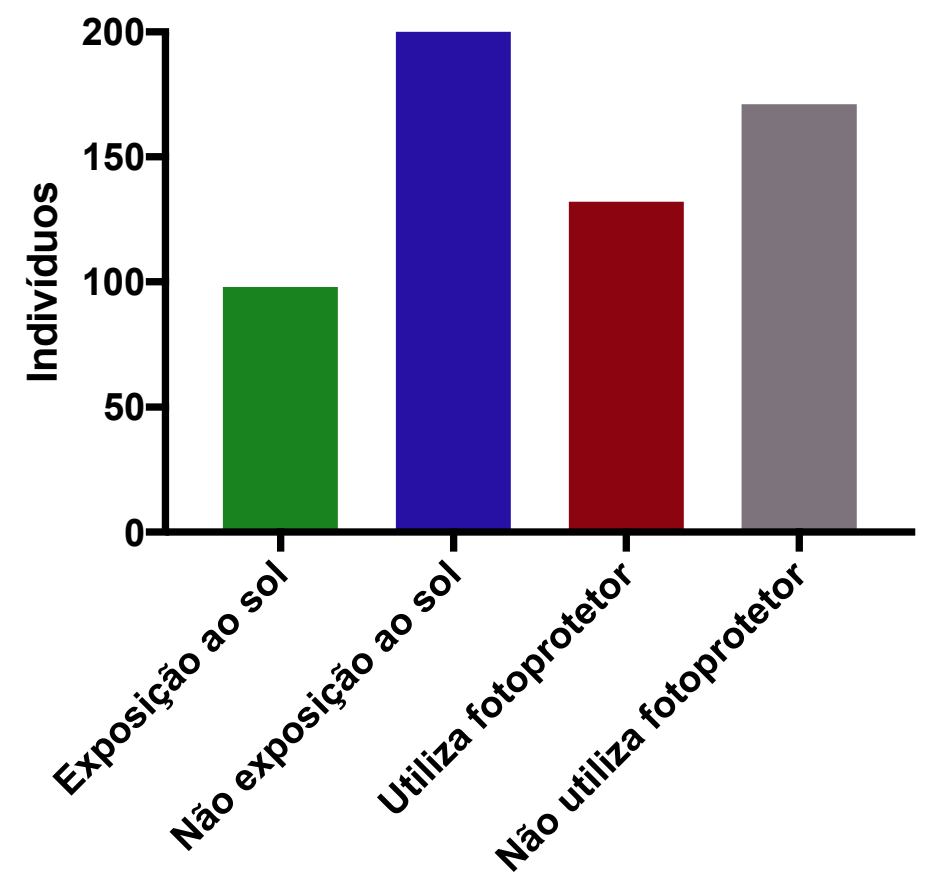

Fonte: Acervo dos autores

\section{Discussão}

Nossos dados demonstraram que há uma baixa aderência ao uso de fotoprotetor, bem como a exposição aos raios solares é frequente na população estudada. Importante mencionar que 136 (45,6\%) indivíduos estudados declararam-se brancos, seguidos de 96 (32,2\%) pardos e $54(18,1 \%)$ declararam-se negros. Sabidamente indivíduos com menor concentração de melanina apresentam uma maior susceptibilidade para o desenvolvimento de cânceres e tumores de pele, especialmente o melanoma (BOCZAR et al., 2019; DE MELO et al., 2018; MAHENDRARAJ et al., 2017). A população do nosso estudo é predominantemente jovem e é importante mencionar que dentre os cânceres mais frequentes em adultos jovens, o melanoma é o segundo mais frequente (BARR et al.,2006).

Um dado importante que foi descrito nesse estudo é que menos de $11 \%$ são tabagistas, esse achado está de acordo com os dados recentes descritos pelo ministério da saúde, que durante o período de 2006 a 2018 tem sido observada uma redução considerável no tabagismo em território nacional (BRASIL, 2012). Os mecanismos patológicos do uso do cigarro tem efeito direto de envelhecimento e estão bem explorados, dessa forma podemos ressaltar uma 
alteração na biossíntese do colágeno (KNUUTINEN et al., 2002), bem como um aumento da produção de elastases por neutrófilos e diversas metaloproteases em virtude da produção exacerbada de espécies reativas ao oxigênio (ROS) (GHOSH et al., 2019), o que substancialmente interfere na integridade da matriz extracelular do tecido conjuntivo. Além disso, o uso constante do cigarro contribui para um aumento da viscosidade sanguínea, o que potencialmente interfere na microcirculação e pode resultar em impactos nutricionais e respiratórios de células em aspectos histológicos (ALKAN et al., 2014). Em conjunto, todos esses tem características fundamentais que contribuem para o aparecimento das características morfológicas ligadas ao envelhecimento cutâneo.

O uso frequente de etanol, por sua vez, esteve presente em cerca de $42 \%$ dos voluntários entrevistados. O uso de etanol está ligado a uma série de mudanças características, como o envelhecimento cutâneo facial, perda de volume facial, perda de vasos sanguíneos e aparecimento de linhas faciais (GOODMAN et al., 2019). Do ponto de vista biológico, o uso do etanol pode representar um papel importante no envelhecimento de uma maneira geral, no entanto, quando consideramos o envelhecimento cutâneo, o etanol pode contribuir para a produção de ROS em nível tecidual, o que fisiopatologicamente auxiliaria em dano tecidual e envelhecimento cutâneo (RINNERTHALER et al., 2015).

Com relação aos aspectos ligados ao sono, grande parte da nossa população alega dormir 6 horas ou menos. Diversos indicadores de qualidade de sono recomendam pelo menos 6 horas de sono como, por exemplo, o Indice de Qualidade de Sono de Pittsburgh. Um estudo que avaliou a capacidade de recuperação da pele após a exposição a luz UV, averiguou que os indivíduos com boas horas de sono apresentavam uma melhor recuperação do que aqueles que não tinham um boa qualidade de sono (OYETAKIN-WHITE et al., 2015). Sendo assim, a frequência de uma baixa qualidade de sono pode representar um importante fator intrínseco para o envelhecimento cutâneo.

Importante destacar que nossos dados, corroboram outros dados de outras regiões brasileiras, onde muitos jovens não fazem uso de foto proteção e se expõem excessivamente ao sol (BATISTA COSTA; BLESSMAN WEBER, 2004). Com relação ao consumo de álcool os nossos dados mostram valores preocupantes e maiores do que o que foram observados a alguns anos atrás (GARCIA; FREITAS, 2015).

Em resumo, nossos dados revelaram uma grande falta de aderência a algumas medidas importantes para a desaceleração do envelhecimento cutâneo, que além de interferir em aspectos estéticos, interfere em aspectos morfológicos e fisiológicos da pele, e pode contribuir com uma menor resistência do tecido, bem como o aparecimento de eventos carcinogênicos. 
Desta forma, torna-se plausível o uso de medidas socioeducativas, a fim de estimular o uso de protetor solar mais frequente, bem como a conscientização com demais aspectos que contribuem para o envelhecimento cutâneo, como o tabagismo, etilismo e aderir as práticas voltadas para uma melhor qualidade de vida.

\section{REFERÊNCIAS}

ALKAN, F. A. et al. The evaluation of plasma viscosity and endothelial dysfunction in smoking individuals. Clin Hemorheol Microcirc., v, 58, n. 3, p. 403-413, 2014.

BARR, R. D. et al. Incidence and incidence trends of the most frequent cancers in adolescent and young adult Americans, including "nonmalignant/noninvasive" tumors. Cancer, v. 122, n. 7, p. 1000-1008, abr. 2016.

BATISTA COSTA, F.; BLESSMAN WEBER M. Avaliação dos hábitos de exposição ao sol e de fotoproteção dos universitários da Região Metropolitana de Porto Alegre, RS. An Bras Dermatol, v. 9, n. 2, p. 149-155, 2004.

BOCZAR, D. et al. Analysis of melanoma in African American patients in the United States. Anticancer Res., v. 39, n. 11, p. 6333-7, 2019

BONTÉ, F. et al. Skin changes during ageing. In: Subcellular biochemistry. Springer New York, 2019. p. 249-80.

BRASIL. Ministério da Saúde. Secretaria de Vigilância em Saúde. Vigitel Brasil 2018: Vigilância de fatores de risco e proteção para doenças crônicas por inquerito telefônico. Brasília, DF: Ministério da Saúde, 2012. 134 p. Disponível em: http://bvsms.saude.gov.br/bvs/publicacoes/vigitel_brasil_2011_fatores_risco_doencas_cronic as.pdf. Acesso em: 10 set. 2020.

DE MELO, A. C. et al. Melanoma signature in Brazil: Epidemiology, incidence, mortality, and trend lessons from a continental mixed population country in the past 15 years. Melanoma Res., v. 28, n. 6, p. 629-636, dez. 2018.

GARCIA, L. P.; FREITAS, L. R. S. Consumo abusivo de álcool no Brasil: resultados da Pesquisa Nacional de Saúde 2013. Epidemiol e Serviços Saúde, v. 4, n. 2), p. 227-237, 2015.

GHOSH, A. et al. Chronic E-cigarette use increases neutrophil elastase and matrix metalloprotease levels in the lung. Am J Respir Crit Care Med, v. 200, n. 11, p. 1392-1401, dez. 2019.

GOODMAN, G. D. et al. Impact of smoking and alcohol use on facial aging in women: Results of a large multinational, multiracial, crosssectional survey. Journal of Clinical and Aesthetic Dermatology, v. 12, p. 28-39, 2019.

KAMMEYER, A.; LUITEN, R. M. Oxidation events and skin aging. Ageing Research Reviews, v. 21, p. 16-29, 2015. 
KNUUTINEN, A. et al. Smoking affects collagen synthesis and extracellular matrix turnover in human skin. Br J Dermatol, v. 146, n. 4, p. 588-594, 2002.

KRUTMANN, J. et al. The skin aging exposome. Journal of Dermatological Science, v. 85, p. 152-161, 2017.

MAHENDRARAJ, K. et al. Malignant Melanoma in African-Americans. Med (United States), v. 96, n. 15, abr. 2017.

OYETAKIN-WHITE, P. et al. Does poor sleep quality affect skin ageing? Clin Exp Dermatol, v. 40, n. 1, p. 17-22, jan. 2015.

RINNERTHALER, M. et al. Oxidative stress in aging human skin. Biomolecules MDPI AG, V. 5, p. 545-589, 2015.

TOBIN, D. J. Introduction to skin aging. J Tissue Viability, v. 26, n. 1, p. 37-46, fev. 2017

YIN, L. et al. Skin aging induced by ultraviolet exposure and tobacco smoking: Evidence from epidemiological and molecular studies. Photodermatol Photoimmunol Photomed, v. 17, n. 4, p. 178-83, 2001.

YOUNG, A. R. Acute effects of UVR on human eyes and skin. Progress in Biophysics and Molecular Biology. Pergamon, 2006. v. 92. p. 80-85.

\section{Como referenciar este artigo}

SANTOS, M. C. O.; ELIZEU, S. O.; MATOS, L. M.; SOARES, G. S.; MACRIS, S. P. C. S.; NALI, L. H. S. Análise da exposição a fatores de risco extrínsecos associados ao envelhecimento da pele em universitários. Temas em Educ. e Saúde, Araraquara, v. 16, n. 2, p. 621-629, jul./dez. 2020. e-ISSN 2526-3471. ISSN 1517-7947. DOI: https://doi.org/10.26673/tes.v16i2.14054

Submetido em: 04/04/2020

Revisões requeridas: $14 / 08 / 2020$

Aprovado em: 20/08/2020

Publicado em: 27/08/2020 